\title{
A Brief Analysis of Honeysuckle Patterns in Wei, Jin, Southern and Northern Dynasties
}

\author{
Xirui Peng \\ ${ }^{1}$ Faculty of Fine Arts, Hunan Normal University, Yuelu District, Changsha, Hunan, China \\ a970552752@qq.com
}

\begin{abstract}
Honeysuckle pattern is the theme of decorative patterns introduced into my country along with Buddhism and Buddhist art. Its appearance means that the central theme decorated with animal patterns in the Han Dynasty and before the Han Dynasty began to be broken. This article mainly studies the social background, the form of the honeysuckle pattern that was popular in the Southern and Northern Dynasties, and the characteristics of tolerance and openness of Chinese culture embodied. The development of the honeysuckle pattern has given us a lot of enlightenment in the developing society. How to inherit the tradition and how to face the infiltration of foreign cultures. The use of the honeysuckle pattern in the design also brings some thoughts.
\end{abstract}

Keywords: Honeysuckle pattern, Northern and Southern Dynasties, Pattern features

\section{浅析魏晋南北朝时期忍冬纹}

\author{
彭西瑞
}

${ }^{1}$ 湖南师范大学美术学院, 岳麓区, 长沙, 湖南, 中国

a970552752@qq.com

\section{摘要}

忍冬纹，是随着佛教和佛教艺术一起传入我国的装饰花纹题材。它的出现意味着汉代以及汉代以前以动物纹 样装饰的中心题材开始被打破。本文主要研究忍冬纹在南北朝时流行的社会背景、呈现的形态以及所体现的中 国文化包容与开放的特性。忍冬纹样的发展给了我们现在正在发展中的社会很多启发, 如何去继承传统, 又如 何去面对外来文化的渗入，忍冬纹样在设计中的运用也带来了一些思考。

关键词: 忍冬纹样, 南北朝, 纹样特征

\section{1. 南北朝时期的社会状态}

三国两晋南北朝是中国历史上长期混战的时代, 它上承两汉, 下启隋唐, 是一种重要的过渡时期。这 一时期的社会经济、文化都遭到了严重的摧残和破坏， 在这个社会动荡的时期, 战争与分裂使得当时中原各 民族的人们被迫迁徙, 受尽苦难, 阶级矛盾与民族矛 盾非常尖锐, 在三百六十多年的时间里, 王朝更迭了 三十余次。

在这种混乱的时代里, “人人厌苦, 家家思乱”, 因此佛教开始兴盛起来, 成为乱世里人们的精神寄托。
正如列宁曾经说过的: “被剥削阶级由于没有力量同 剥削阶级进行斗争，必然产生对死后幸福的生活的憧 憬。”因而佛教宣扬的“生死轮回”，“因果报应”正 是在诱骗人们不要绝望现世的苦难与痛苦, 而要学会 把今生的苦难寄托在来生的福报上。与此同时战争增 加了各民族文化相互交融的机会。在这一时期的中国 虽然处于混战时期，但是从西汉时期开始的丝绸之路 并没有因此断了，使这一时期中国文化的发展呈现出 一种特别的状态，中原内地南北文化相互交融，中西 方通过丝绸之路佛教开始在中国逐渐兴盛、以及波斯、 希腊文化的影响, 而忍冬纹则是在这复杂的社会背景 下，发展成为南北朝运用得最多的植物纹样。 
忍冬花的特性与佛教所宣扬的精神高度契合, 因 此被作为一种寓意吉祥的纹样运用到佛教艺术的各 个地方。

\section{2. 忍冬纹装饰的源流}

“金花间银莣, 清雅香怡人。”这是金代诗人赞美 忍冬的诗句, 说忍冬可能不知道是何物, 但另一个名 字, 一定有所耳闻, 它的俗名又叫 “金银花”, 为一种 蔓生植物, 通称卷草。其花瓣垂须, 黄白相半, 故名 金银花，又因越冬不调而得 “忍冬”。

“金银花”,也是一味具有悠久历史的中药, 曾被 李时珍收录于他的《本草纲目》中，它因为凌冬而不 调而得名。忍冬纹是随着佛教和佛教艺术一起传入中 国的, 作为外来纹样, 忍冬纹在东汉末年就已经开始 出现, 在魏晋南北朝时最为流行, 莲花纹和忍冬纹成 为最具有时代特色的纹样, 广泛地出现在绘画、石窟 和织物等艺术品的装饰上, 佛教艺术上也大量地运用 忍冬纹进行装饰。可以看出, 那时佛教艺术装饰题材 的重要影响, 几乎触及人们生活用具的各个方面。

追根溯源, 忍冬纹它是起源于古埃及的莲花和纸 莎草, 在希腊通常以棕榈叶、莨苔叶为主要题材, 将其 融合而形成的卷草原型, 随后传入罗马, 随着罗马帝 国的东征将卷草纹样带到了恒河流域, 而后再传到中 东地区、印度和中国, 背景、地域以及文化都不尽相同 的情况下形成了具有不同特征的卷草纹类型。忍冬纹 样是中国工匠在传统装饰纹样云气纹的基础上结合 外来文化的卷草纹, 而后再创造通过古代人们的创造, 从而形成了完全不同于其它地域的具有中国民族文 化气息的植物装饰纹样式。

忍冬纹常以叶状植物纹样的形式, 组成波曲线状 结构的枝干, 一个单元的枝干上一般有三个叶片, 和 一个相对的叶片, 其变化多种多样, 有单叶, 有双叶; 有两页顺向，也有两页相背、以及倒顺等多种形态。 在南北朝时期它被广泛地运用在佛教艺术、墓葬器物、 刺绣、陶器等地方。忍冬是中国古代装饰中最早出现 的植物纹样之一, 它在魏晋南北朝开始大量流行, 这 意味着从商周开始的以动物以及神兽云气纹样做为 中心的装饰题材的局面已经开始被打破, 从魏晋南北 朝开始到隋唐以后开始逐渐进入到以植物纹样为中 心的新的历史阶段。

\section{3. 南北朝时期忍冬纹样的特征}

南北朝时期, 忍冬纹其叶瓣形态有单叶、双叶、 多叶等多种, 作为一种边饰, 它主要被应用在石窟寺、 墓葬、器物等装饰之上, 主要是以单独纹样、适合纹 样、二方连续纹样的形式出现的形式居多。

这种看似很简单的植物纹样题材, 却被古代中国 的工匠们演绎得丰富多彩。忍冬纹虽是外来纹样, 但 它在发展的过程当中, 却被工匠们融入了中国文化的 气息和审美，使之具有中国的作风和气派。在南北朝
时期的忍冬纹样主要呈现出一种清瘦、程式化的风格 特征。以下将从特征及应用范围两方面分析。

\section{1 清瘦、程式化特征}

魏晋南北朝时期的忍冬纹比较清瘦和程式化, 一 般以三叶为一个基本的单元，主结构线以 $\mathrm{S}$ 型作为主 结构线, 这就使得纹样的运用变得更加灵活, 从而被 广泛地运用在各种装饰物上，而这也对此后的纹样、 图案的发展产生了重要的影响。这一时期的忍冬纹样 主要是以单独纹样、适合纹样、二方连续纹样的形式 出现的形式居多。单独忍冬纹样又可分为对称式与自 由式 (即不对称样式); 适合纹样以圆形适合纹样、方 形适合纹样为主; 二方连续则是将一个单位的忍冬纹 样，向上下或者左右延展连续成一条带状式纹样，忍 冬纹的二方连续纹样的形式主要有波形、桃形、缠枝 等等形态。这是对南北朝的忍冬纹的程式化形态的大 分类, 它丰富的形态变化与当时的文化状态有着紧密 的联系，忍冬纹样的变化过程当中，也体现了中华民 族思想的逐渐变化, 以及中华文化包容的特性。

清瘦、程式化的忍冬纹具有它独特的审美，它大 多的二方连续形态使得它具有装饰的节奏感和韵律 感。在连续的曲线骨架上有着枝叶的起伏和朝向, 主 枝干与小叶曼之间大小波曲线此起彼伏, 形成很好的 节奏感。在作为边饰的同时, 具有细节, 却也流畅, 更加能烘托主题装饰的氛围。

\section{2 应用范围}

忍冬纹在佛教艺术中有着十分吉祥的象征意义, 这决定了它与佛教的关系非常密切, 所以忍冬纹在 魏晋南北朝时期的佛教石窟艺术中被大量地运用, 它通常被广泛的应用于石窟建筑中的各个部位, 例如 佛光、石碑两侧以及藻井等地方。

佛教的宗教色彩除了魏晋南北朝时期地石窟上 有突出体现, 在陶罐、墓葬、以及染织物上都有所体 现。笔者主要从石窟、墓葬中的画像砖以及织物上来 阐述忍冬纹在这些方面的具体体现。

\section{2.1 石窟}

魏晋南北朝时期, 石窟佛旣閶椐有着大量的忍冬 纹样。佛拿是指在岩崖上丵洞, 再一个个的洞里放置 佛像、菩萨。在敦煌石窟中大部分佛拿龛门的顶上都 呈拱形从而来装饰石窟壁面，壁面通常采用彩绘或 是浅浮雕的形式来进行装饰。在敦煌石窟中, 最典型 的毞椐样式为尖拱形龛, 忍冬纹通常从拿额两端向 下垂, 呈翻卷状态。芘硘额端内的装饰以忍冬纹为主, 中间穿插莲花、童子或着珍禽异兽、伎乐天人、飞马 等装饰纹样。

石窟建筑的顶部装饰，如平棋、藻井和人字坡部 位也大量运用了忍冬纹作为装饰图案。平棋是天花的 别称，略同于藻井，结构形同方格状棋盘，层层叠 
叠，每一个方格为一井，装饰以雕刻、绘画等，呈连 续排列的方格形装饰。为大部分分布于中心柱的四周 石窟顶，每个方格内装饰大都采用重重套叠的形式， 其装饰主要是以莲花纹样为中心，每层以忍冬纹为 边饰。装饰常见的内容有忍冬、莲花、飞天、仙佛、 天马等等。

藻井装饰非常多样化, 绘制精致。敦煌石窟的藻 井是在中国古代传统的建筑层叠的屋顶做了简化处 理。中间的方格用作装饰题材的主题，其余方格放射 状，外围一层层的方格逐步将主题细化。中心的装饰 大部分会用垂莲, 层层方格的边框多饰以连续的忍 冬纹或其他花卉图案, 角处饰以忍冬、火焰、莲花、 飞天等适合纹样。忍冬纹样这样大量地在石窟中使用, 一是因为它可以将装饰的主题有连贯性, 但又不会使 画面僵化, 二是因为它本身的吉祥含义与佛教艺术十 分契合。在做边饰的同时也烘托出了一种给人吉祥、 安定的感觉。

人字坡是指石窟仿木结构的屋顶,一般位于石窟 内部的顶端位置, 而为了适应人字坡这种特殊的石窟 顶结构, 敦煌石窟中产生了一种独特的装饰图案, 即 所谓的 “人字坡图案”。敦煌莫高窟的人字坡装饰大 多运用仙佛、飞天妓乐、吉祥植物等装饰题材, 常见 的植物纹样有忍冬纹、莲花, 这些纹样一般穿插在有 飞天人物、仙佛等内容之中。也有大量运用单独的忍 冬纹样的形式来装饰, 使得整体纹样更加丰富, 且具 有流畅感。

忍冬纹, 在石窟装饰中主要用二方连续的形式 出现起到边饰的作用, 从而形成具有规律的装饰节奏 感。边角装饰是最具有形式美感的部分, 也是出现次 数最多、变化最丰富的部分。早期的忍冬纹具有比较 浓郁的异域特征, 越到后期, 它开始被工匠运用熟练, 开始加入中国古代文化、思想以及审美于其中, 忍冬 的形制开始更加自由, 寓意也加入了古代人们的美好 憧憬。

\subsection{2 画像砖}

在秦汉时期, 重视厚葬, 人们 “视死如视生” 观 念深入人心, 当然重视厚葬的观念主要针对的是王公 贵冒之家，普通民众在封建社会里活着已是不易。 1972 年在长沙马王堆考古人员发现的辛追夫人墓就 是典型的 “千金之家”, 当时的重墓葬以及神仙思想的 弥漫可见一斑。这之后的六朝, 尤其是南北朝时期, 重墓葬思想开始慢慢地淡化, 因为战乱的现实条件也 不允许, 但是在魏晋南北朝时期墓葬中仍然会发现画 像石画像砖的使用, 希望死后也能过的很好的夙愿是 每个朝代人们都相似的地方。

魏晋南北朝时期的画像砖继承了汉代了艺术传 统, 同时也渗入了佛教的宗教色彩。装饰主题除了有 汉代车马出行、奇珍异兽之外, 开始出现莲花、忍冬、 山石树木也开始出现, 并逐渐占据装饰题材的重要位 置, 因为采用波曲的忍冬纹样和部分莲花纹, 使得它
不同于汉代的古拙质朴的风格, 更多的是一种清秀雅 致的艺术特征。以同时也体现了以动物为中心的装饰 题材开始逐渐向以植物为中心的题材过渡, 也体现了 人们开始关注现世生活的一花一木, 与自己生活息息 相关的事物开始走进装饰的题材中。

\section{2.3 织物}

魏晋南北朝时期的染织工艺也非常地发达, 其中 以四川蜀锦最为出名, 蜀锦甚至是三国时期魏国和吴 国所争相追求的上等丝织物。但是六朝丝织物存世的 资料非常少，这和当时战乱的社会背景以及现今所发 现的考古资料有限相关。

1965 年, 在敦煌莫高窟 125-126 窟中曾发现了一 些北魏时期的刺绣残品, 一佛二菩萨说法图, 织物的 花边上四片忍冬纹组成的圆圈纹，纹样用浅黄、蓝绿 等颜色与其他纹样组合后构成了一幅富有变化的几 何纹样图案。这种丝织物的纹样图案在继承汉代云气 纹样的基础上又有所发展, 不同于汉代云气纹的不规 则变化的形式, 忍冬纹是由有规则的波形骨架构成, 更加地规律化, 几何化。

\section{4. 结论}

在魏晋南北朝这样一个十分动乱的时代里, 因为 人们现世的痛苦无法解决只好寄托于来生的思想, 使 得佛教在这个苦难的年代里迅速地发展起来, 因此人 们的思想受到了佛教很大的影响, 带有浓厚的宗教色 彩, 同时也受到本土文化中玄学的影响, 整体的思想 上偏宗教化, 而这也正是魏晋南北朝时期的突出特征。

流行于魏晋南北朝时期的忍冬纹样, 由外域传来 之时带有浓厚的异域气息, 但是在经过中国工匠的艺 术处理, 它呈现出一种中国气息以及中华民族独有的 审美。忍冬纹到后来结合莲花纹、云纹等其他一些当 时流行的植物装饰纹样形成新的装饰纹样——卷草 纹, 后忍冬纹及卷草纹常常与其他纹样相结合, 在家 具、建筑等方面广为应用。忍冬, 能够越冬不死, 所 以被人们用来象征人的灵魂不灭、轮回永生, 忍冬这 种象征奇迹、不死、长生的药物, 对于追求长生不老 的修道者来说是一种十分宝贵的宝物。也形成了一种 固定的文化符号, 不仅美观, 而且还蕴含着生死轮回 的美好寓意。

忍冬纹样传入、变化、最后消融在中国文化中称 为之后的唐卷草等纹样, 深刻说明了中华文化所特有 的包容性, 在受外来文化影响的同时始终有自己的性 格, 最后能将外来文化吸纳归为自己所用。这也给了 我们现在正在发展中的社会很多启发, 如何去继承传 统, 又如何去面对外来文化的渗入, 忍冬纹样的发展 给我们提供了这样的一个思路与启发。

在设计中则更需要这样不断吸纳、融合、输出的 一个过程, 只有真正为自己所吸收, 才会更好地做出 好的设计, 只有不断地发展才能不被时代所抛弃, 这 
就是忍冬纹发展的路径给我们最大的启发吧。

\section{REFERENCES}

[1] Ni, J.L. (2004) From honeysuckle to curly grass pattern. Decoration, 12.

[2] Qian, Y.R. (2016) A study of honeysuckle patterns in Dunhuang murals. Beijing Forestry University.

[3] Wu, S. (1992) Chinese decorative patterns in past dynasties (3). People's Fine Arts Publishing House, Beijing.

[4] Jiang, B.L. (2004) Research on the History of Chinese Zoroastrian Art. Sanlian Bookstore, Beijing.

[5] Tian, Z.B. (2010) History of Chinese Arts and Crafts. Oriental Publishing Center, Shanghai. 